\title{
Calendar of Forthcoming Meetings Powder Diffraction March 2014
}

\author{
Gang Wang \\ Research and Development Center for Functional Crystals, Institute of Physics, Chinese Academy of Sciences, \\ No.8 Nansanjie, Zhongguancun, Haidian District, Beijing 100190, China \\ gangwang@iphy.ac.cn
}

\section{1-25 April 2014}

2014 MRS Spring Meeting \& Exhibit San Francisco, California, USA [Info: http://www.mrs.org/spring2014/]

\section{1-16 May 2014}

11th International Conference on Quasielastic Neutron Scattering (QENS 2014) L'Escandille, Autrans, France [Info: http://www.ill.eu/news-events/events/qens-2014-wins-2014/]

\section{2-15 May 2014}

2014 APS/CNM/EMC Users Meeting Argonne, Illinois, USA [Info: http://usersmeeting2014.conference.anl.gov/]

\section{4-28 May 2014}

2014 Annual Meeting of the ACA (American Crystallographic Association) Albuquerque, New Mexico, USA [Info: http://www.amercrystalassn.org/content/pages/ main-annual-meetings]

\section{5-18 June 2014}

14th European Powder Diffraction Conference (EPDIC-14) Aarhus, Denmark [Info: http://epdic.ing.unitn. it/html/epdic_14.html]

\section{7-11 July 2014}

International Conference on Surface X-Ray and Neutron Scattering Hamburg, Germany [Info: http://www.sxns13.de/]

\section{1-25 July 2014}

11th International Conference on the Structure of Surfaces (ICSOS-11) University of Warwick, Coventry, UK [Info: https://www.eventsforce.net/iop/frontend/reg/ thome.csp?pageID=106741\&eventID=264\&eventID=264]

28 July-1 August 2014

Denver X-ray Conference: 63rd Annual Conference on Applications of X-ray Analysis Big Sky, Montana, USA [Info: http://www.dxcicdd.com/]

\section{3-7 August 2014}

The International Union of Microbeam Analysis Societies Quadrennial Meeting Hartford, Connecticut, USA [Info: http://iumas6.org/]

\section{5-12 August 2014}

23rd Congress and General Assembly of the International Union of Crystallography (IUCr2014) Montreal, Quebec, Canada [Info: http://iucr2014.org]

\section{5-29 August 2014}

FEL 2014 -Free Electron Laser Conference Basel, Switzerland [Info: http://www.fel2014.ch/]

14-19 September 2014

12th Biennal Conference on High-Resolution X-Ray Diffraction and Imaging (XTOP 2014) Grenoble \& Villard-de-Lans, France [Info: http://xtop2014.org/]

\section{7-20 September 2014}

15th International Conference on the Crystallisation of Biological Macromolecules (ICCBM 15) Hamburg, Germany [Info: http://www.iccbm15.org/]

21-23 September 2014

German Conference for Research with Synchrotron Radiation, Neutrons and Ion Beams at Large Facilities 2014 Bonn, Germany [Info: http://sni-portal.uni-kiel.de/ sni2014/]

\section{3-25 September 2014}

MSE 2014 Darmstadt, Germany [Info: http://www.dgm.de/ dgm/mse-congress/]

\section{5-10 October 2014}

10th World Conference on Neutron Radiography (WCNR-10) Grindelwald, Switzerland [Info: http://indico. psi.ch/conferenceDisplay.py?confId=2019]

26-31 October 2014

12th International Conference on X-ray Microscopy (XRM 2014) Melbourne Convention and Exhibition Centre, Melbourne, Australia [Info: http://www.xrm2014.com/]

30 November-5 December 2014

2014 MRS Fall Meeting \& Exhibit Boston, Massachusetts, USA [Info: http://www.mrs.org/fall2014/]

\section{3-28 August 2015}

The 29th European Crystallographic Meeting Rovinj, Croatia [Info: http://ecm29.ecanews.org/]

13-18 September 2015

16th International Conference on Small-Angle Scattering Berlin, Germany [Info: http://www.helmholtz-berlin.de/ events/sas/] 